\title{
Urinary Tract Infection, CTCAE
}

National Cancer Institute

\section{Source}

National Cancer Institute. Urinary Tract Infection, CT CAE. NCI Thesaurus. Code C143904.

A disorder characterized by an infectious process involving the urinary tract, most commonly the bladder and the urethra. 